\title{
OPERATIONS OF THE LEDA RESONANTLY COUPLED RFQ*
}

\author{
L. M. Young ${ }^{\dagger}$, Los Alamos National Laboratory, Los Alamos, New Mexico 87545
}

\begin{abstract}
The LEDA RFQ is a $350-\mathrm{MHz}$ continuous-wave (CW) radio-frequency quadrupole linac. LEDA is the low energy demonstration accelerator, a full power front-end prototype for the accelerator production of tritium (APT) linac. This machine has accelerated a $100-\mathrm{mA} \mathrm{CW}$ proton beam from $75 \mathrm{keV}$ to $6.7 \mathrm{MeV}$. The 8-m-long RFQ accepts a dc, $75-\mathrm{keV}, \sim 110-\mathrm{mA} \mathrm{H}^{+}$beam from the LEDA injector, bunches the beam, and accelerates it to full energy with $\sim 94 \%$ transmission. Output beam power is $670 \mathrm{~kW}$. This RFQ consists of four 2-meter-long RFQs joined with resonant coupling to form an 8-meter-long RFQ. The resonant coupling improves the stability of both the longitudinal and transverse RF-field distribution in this long RFQ.
\end{abstract}

\section{RFQ DESCRIPTION}

The RFQ [1-5] receives a continuous stream of $75-\mathrm{keV}$ protons from the $\mathrm{H}^{+}$injector, [6,7] forms it into bunches with a high capture efficiency ( 94\%), and then accelerates these bunches to an energy of $6.7 \mathrm{MeV}$. The RFQ consists of an 8-m-long cylindrical resonant cavity excited in the TE21 mode, inside which four scalloped vanes are mounted in an orthogonal array about the axis. These vanes provide the necessary transverse and longitudinal RF electric fields to simultaneously accelerate and focus the beam. The cavity is constructed in four resonantly coupled, 2-m-long copper sections that are water cooled by multiple internal channels to remove RF heating and provide frequency control. Figure 1 shows the coupled RFQ structure mounted in the tuning laboratory. Figure 2 shows the RFQ structure configuration including tapered RF power feeds, vacuumport placement, and section nomenclature. Figure 3 shows a photograph of the completed RFQ assembly in the LEDA tunnel with the injector pulled back. The array of vacuum manifolds, water-cooling manifolds, and $\mathrm{RF}$ waveguide almost completely hides the accelerating structure.

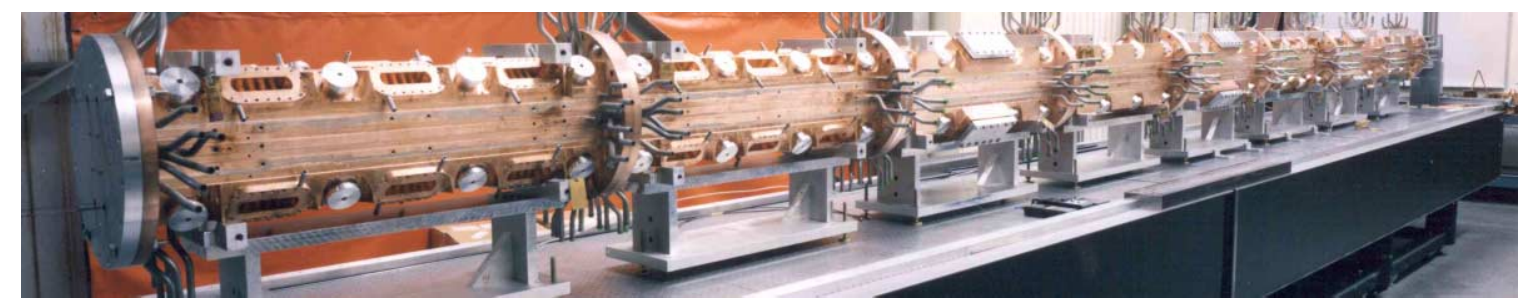

Figure 1: Eight-meter-long RFQ in the tuning laboratory. The adjustable slug tuners can be seen in this picture.

\footnotetext{
* Work supported by the US DOE, Defense Programs.

†lyoung@lanl.gov
}

\subsection{Design features}

With output energy of $6.7 \mathrm{MeV}$ the LEDA RFQ $[1,8]$ is the highest energy and highest power RFQ in the world $[3,5,9-11]$. The beam power is $670 \mathrm{~kW}$ when operated with the design-value $100-\mathrm{mA} \mathrm{CW}$ proton beam, making it the second-most powerful operating linear accelerator (after the LANSCE $800-\mathrm{MeV}$ linac). Some of its unique design features are as follows:

- It is the longest 4-vane RFQ in the world. (8 meters)

- It employs resonant coupling $[12,13]$ between the four 2-m-long segments, providing high RF field stability throughout the entire structure length.

- It has a significantly larger aperture and gap voltage in the accelerating section than previously designed RFQs at this frequency.

- Transverse focusing strength at the RFQ entrance is reduced for easier beam injection. This allows placement of the final focusing solenoid in the low energy beam transport (LEBT) at the optimum distance from the RFQ for input matching.

- Transverse focusing at the RFQ exit is reduced to match the focusing strength in the next accelerating structure, the CCDTL [14].

- $\quad$ RF power from three 1-MW klystrons is coupled to the RFQ through six waveguide irises. The structure itself combines the RF power.

\section{RESONANT COUPLING}

In a typical RFQ that has constant focusing strength and constant gap voltage, as vane modulation increases to accelerate the beam, the aperture shrinks and beam can be lost on the vane tips. As the energy rises the cell length increases, and for a given modulation, the accelerating gradient decreases inversely with cell length. Since the maximum practical modulation is about 2, the RFQ would become very long if the gap voltage remained constant. To reduce beam loss and shorten the RFQ, we maintain a 


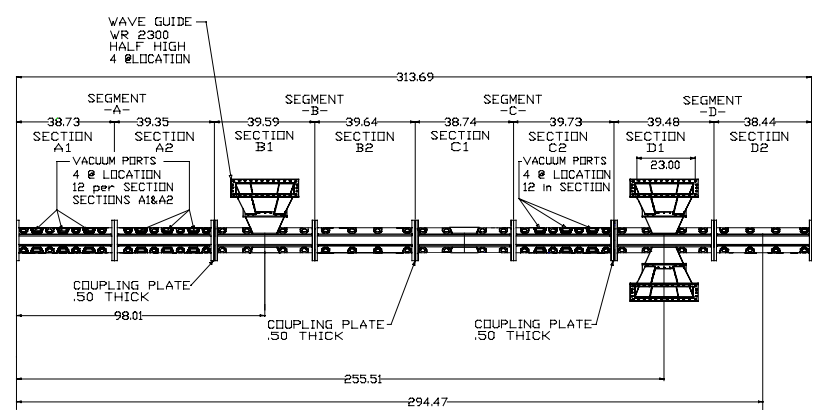

Figure 2: RFQ layout, showing RF feeds, vacuum ports, and segment nomenclature.

large aperture, and increase the vane voltage to partially counter the decrease in transverse focusing as the vane modulation increases. The increased gap voltage substantially increases the accelerating field, thus shortening the RFQ. However, even with this increased gap voltage, eight meters of length is required to accelerate the beam to $6.7 \mathrm{MeV}$. A conventional 8-mlong, $350-\mathrm{MHz}$ RFQ would not be stable. Small perturbations would distort the field distribution intolerably $[12,13]$. Therefore, four 2-m-long RFQs (labeled as segments A, B, C and D in Figure 2) are resonantly coupled to form the 8-m-long LEDA RFQ. The resonant coupling is implemented by separating the four 2-m RFQs by coupling plates. An axial hole in the coupling plates allows the vane tips to nearly touch. The capacitance between the vane tips of one RFQ and the next provides the RF coupling between the 2-m-long segments. The gap between the vane tips at the coupling joint is $0.3175 \mathrm{~cm}$. To minimize the effect of this gap on the beam, the gap position corresponds to a zero crossing of the RF electric field when the bunch passes the gap. The RF field is in phase in all four segments. The "coupling mode" has a strong electric field across the $0.3175-\mathrm{cm}$ gap and has one longitudinal node in each $2-\mathrm{m}$ RFQ. The coupling mode's longitudinal component of electric field transmits RF power, providing the field stability. Adjusting the gap between vane tips tunes the coupling-mode frequency.

\section{RFQ TUNING}

Figure 4 shows a measurement of the fields in the RFQ. The fields were measured with the bead-perturbation technique in the magnetic field region. For this measurement a metal bead rides on a plastic tape that is supported at the ends of the RFQ and at the coupling plates. The plastic tape with the bead moves on a pulley system and travels through all 4 quadrants of the RFQ. The bead perturbs the frequency of the RFQ in proportion to the magnetic-field energy displaced by the bead. The frequency perturbation is measured versus bead position and the relative magnetic field strength is derived. In Figure 4, the bumps in the field are caused by local perturbations in the magnetic field near the slug tuners, which tune the RFQ to the correct field distribution and frequency [15]. The larger dips in the quadrupole magnetic field, that occur every $200-\mathrm{cm}$, are caused by the

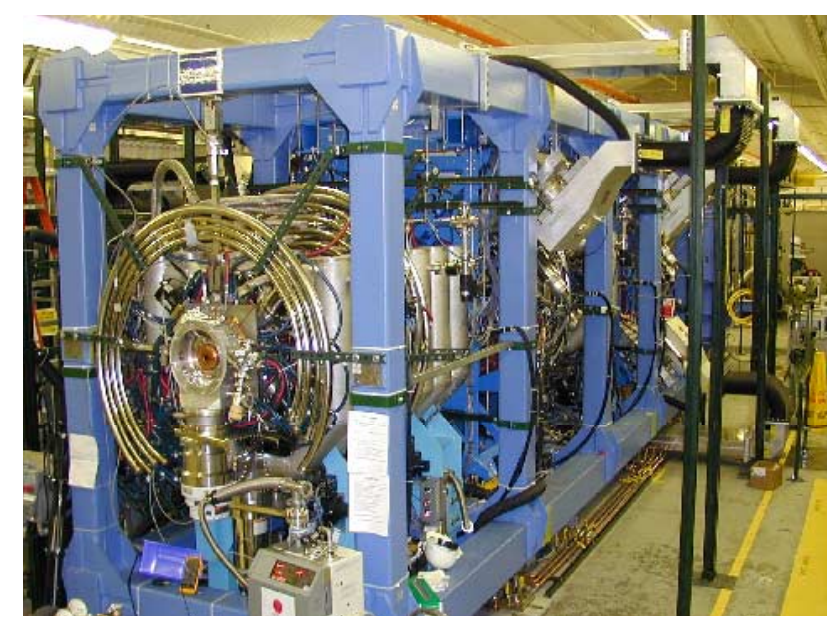

Figure 3: A photograph of the RFQ mounted in the support/alignment frame, looking along the beam direction. The accelerating structure is buried within the array of water-cooling manifolds, vacuum manifolds, and RF waveguide.

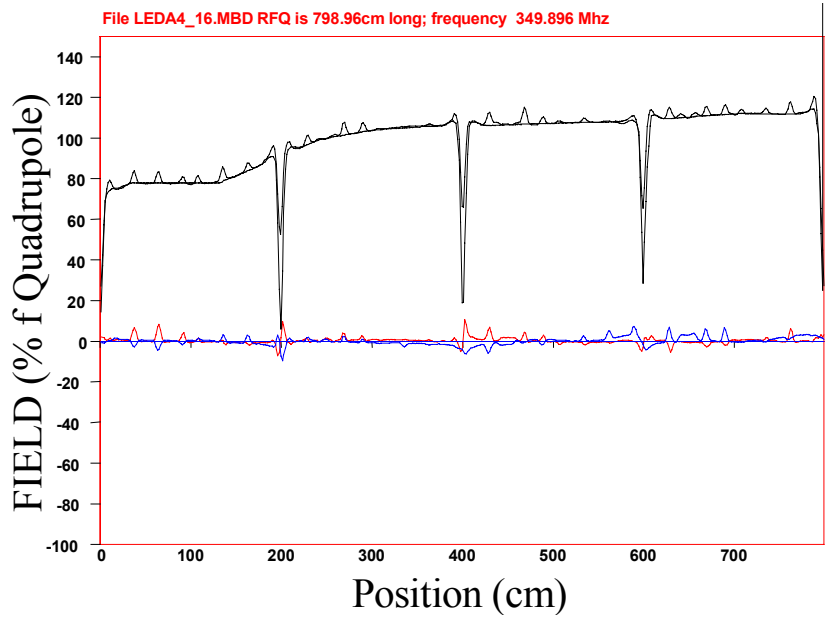

Figure 4: Bead-perturbation measurement in the primarily magnetic field region of the RFQ. The quadrupole magnetic field is normalized to $100 \%$. The two residual dipole modes mixed with the RFQ fields are typically less then $2 \%$. The quadrupole is the sum of the fields in all 4 quadrants. The dipole field strength is proportional to the difference between the fields in opposite quadrants. Also shown is a smooth line that lies on top of the measured quadrupole mode that shows the design value of the magnetic field.

coupling plates. These dips and bumps do not appear in the electric field on axis. Temporary adjustable slug tuners distributed along the outer walls were used to tune the RFQ. The program RFQTUNE, using a least squares fitting procedure, was used to determine the length of these tuners. This program uses the measured field distribution to calculate a set of new tuner penetrations. The new set of tuner penetrations should change the field distribution to match the desired field distribution. After several iterations the field distribution closely matches the desired distribution and the permanent tuners are machined to length when the final tuning is complete. 


\section{RFQ ELECTROMAGNETIC DESIGN}

The RFQ was designed with the code PARMTEQM (Phase and Radial Motion in Transverse Electric Quadrupole; Multipoles) [16]. The code includes the effect of higher-order multipoles in the RFQ fields, which are important in accurately predicting beam loss. The earlier code version, PARMTEQ, used only the first two terms in the expansion of the RFQ fields. PARMTEQM uses the first eight terms. In addition, PARMTEQM requires a realistic description of the input beam to accurately simulate beam losses in the RFQ when the input beam is not ideal. Simulations of the beam transport through the LEBT [17] with PARMELA (Phase and Radial Motion in Electron Linear Accelerators) $[18,19]$ produce a more realistic distribution of particles for input into the RFQ simulation code than the ideal input distributions generated internally by PARMTEQM.

\subsection{RFQ entrance}

To implement the reduced focusing strength at the entrance of the RFQ and have adequate focusing in the interior of the RFQ, the transverse focusing parameter increases smoothly from 3.088 to 6.981 over the first 32 $\mathrm{cm}$ of the RFQ. The focusing parameter is proportional to $V / r_{0}^{2}$ where $V$ is the voltage between adjacent vane tips and $r_{0}$ is the average aperture. The voltage is held constant in this region and the aperture is reduced to increase the focusing parameter. On entry, the beam is not yet bunched, allowing the use of weak transverse focusing. By the time the beam starts to bunch, the focusing is strong enough to confine the bunched beam.

The low focusing strength at the RFQ entrance means that the matched beam size is relatively large, allowing a relatively large distance between the second LEBT solenoid and the RFQ entrance. Without this feature, the solenoid would be right at the RFQ entrance. When space-charge neutralization is taken into consideration, the optimum location for the solenoid is about $15 \mathrm{~cm}$ upstream.

\subsection{Key parameters}

Figure 5 shows some of the key parameters of the RFQ design as a function of position along the structure. These include the aperture, vane modulation, gap voltage, accelerating field, synchronous phase, transverse and longitudinal current limits, and beam energy. Some labels include units: The aperture (labeled "a"), is smallest at the end of the gentle buncher, about $140 \mathrm{~cm}$ into the structure. This is where the transverse current limit is a minimum. Typically, the end of the gentle buncher is the RFQ choke point, which determines the maximum current that can be accelerated $[3,20]$. This calculation of the choke current uses simplifying approximations that yields current limits roughly twice what can be expected from the actual RFQ.

The vane modulation (labeled " $m$ ") has a maximum value just slightly greater than 2 . The gap voltage (labeled "V") ranges from $67 \mathrm{kV}$ to $117 \mathrm{kV}$. The accelerating field (labeled "ez") reaches a maximum value of $1.75 \mathrm{MV} / \mathrm{m}$.

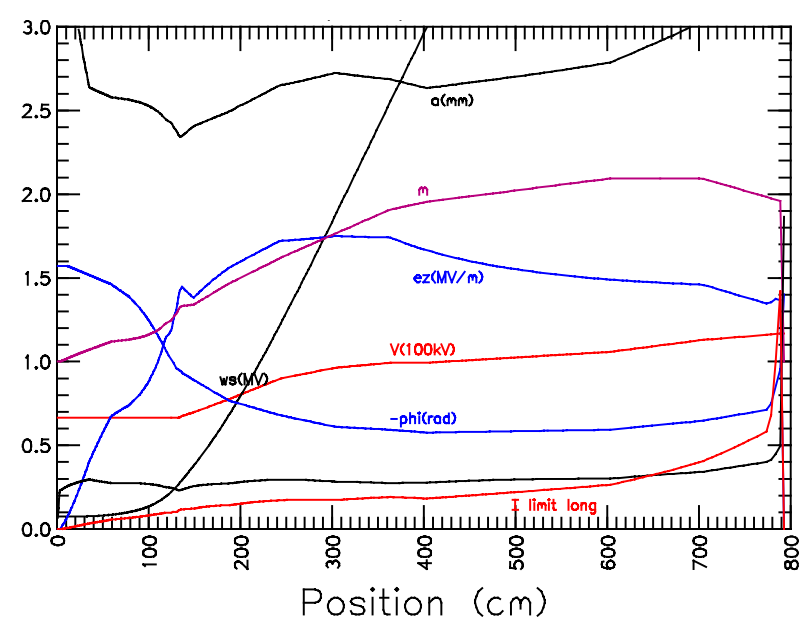

Figure 5: Plot of key RFQ parameters as a function of distance along the structure.

The synchronous phase (labeled "-phi") is plotted in radians and ranges from $-90^{\circ}$ at the RFQ entrance to $-33^{\circ}$ degrees at the midpoint. PARMTEQM predicts values of the current limits. The figure plots the transverse ("I limit tran") and longitudinal ("I limit long") current limits in Amperes. The beam energy (labeled "ws") is plotted in $\mathrm{MeV}$ and goes off scale near the $400-\mathrm{cm}$ point, but continues in a nearly straight line to $6.7 \mathrm{MeV}$ at the end of the RFQ. The peak surface electric field is nearly constant over the whole length of the RFQ at $33 \mathrm{MV} / \mathrm{m}(1.8$ Kilpatrick) [21].

Simulations of the RFQ with PARMTEQM predict normalized transverse-output-beam emittances in the range 0.16 to $0.22 \mathrm{~mm}$-mrad, depending on the input distribution. The simulations also predict the $\mathrm{x}$ and $\mathrm{y}$ emittances to be the same. Initial measurements of these emittance values are 0.25 and $0.31 \mathrm{~mm}-\mathrm{mrad}$ respectively $[22,23]$. There are also minor differences between the predicted and measured Twiss parameters.

\section{X-RAY END POINT MEASUREMENT}

The voltage between adjacent vanes in the RFQ can be determined by measuring the energy of the $\mathrm{x}$-rays emitted from the RFQ, and noting that the maximum X-ray energy corresponds to the vane-to-vane voltage [24]

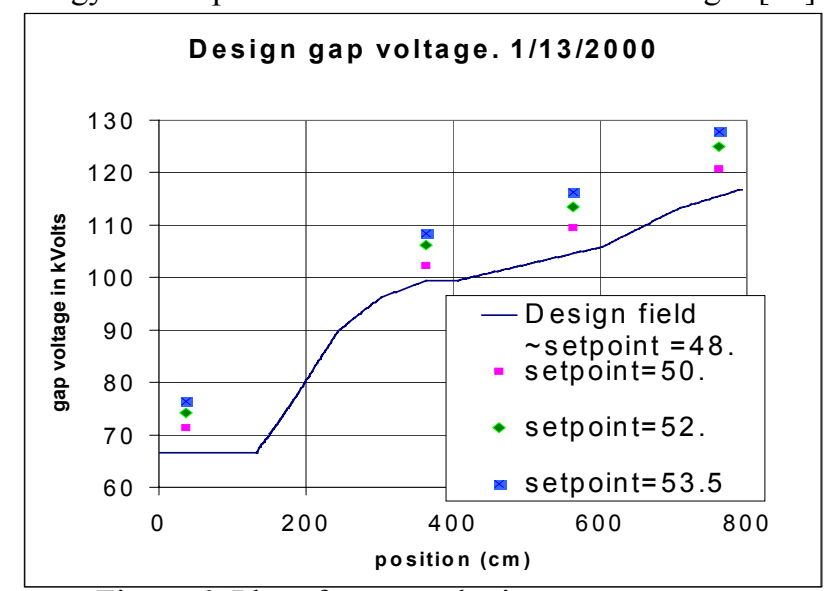

Figure 6: Plot of x-ray endpoint measurements. 
Field-emitted electrons cross this gap in a small fraction of a RF period and, therefore, can gain energy very nearly equivalent to the full gap voltage. Thus, the endpoint of the $\mathrm{x}$-ray spectrum (in $\mathrm{keV}$ ) produced by bremsstrahlung when the electrons strike the opposite vane tip is a measure of the gap voltage in $\mathrm{kV}$. Figure 6 shows a summary of x-ray endpoint measurements at the 4 locations of the RFQ x-ray windows.

\section{TRANSMISSION THROUGH THE RFQ}

The code PARMELA simulates the LEBT beam with 98\% space-charge neutralization [25], except for the last $10 \mathrm{~cm}$ directly upstream of the RFQ, where it ramped down from $98 \%$ to 0 . This simulation showed that the original LEBT could match the beam to the RFQ. However, improved simulations showed that proper matching would be possible only with an electron trap at the RFQ entrance, and a reduced solenoid-to-RFQ distance. $(15 \mathrm{~cm}$ instead of $30 \mathrm{~cm})$. The electron trap is a metal ring at the entrance of the RFQ. A ring voltage of $-1 \mathrm{kV}$ blocks low-energy plasma electrons, but does not affect the $75-\mathrm{keV}$ protons. The electron trap performs two essential functions. One, it improves the space charge neutralization in the LEBT. Two, it prevents electrons from streaming into the RFQ through the beam current torrid and corrupting the measurement of the input beam current.

Using the PARMELA simulated beam from the modified LEBT, three RFQ codes predict $93 \%$ transmission with the RFQ operating at design field levels. The codes are PARMTEQM, which uses 2D space charge effects and RFQTRAK [26] and TOUTITIS [27], which use 3D space and image charge effects. The measured transmission has been as high as $94 \%$ at $100 \mathrm{~mA}$ when the RFQ fields are $10 \%$ above the design field strength. Figure 7 shows a comparison between the calculated and measured transmission as a function of RFQ field strength. The figure also shows an anomalous drop in transmission at the end of a 500- $\mu$ s-long pulse when the RFQ field strength is at or below the design field strength. As shown in Figure 8, there is no drop in transmission observed for 70-mA RFQ output beam currents: as the current increases from 80 to $100 \mathrm{~mA}$, the RF field level at which the drop occurs increases slightly. This effect is discussed in the next section.

\section{POSSIBLE ION TRAPPING IN RFQ}

Figure 9 shows the time dependence of RFQ transmission in a 300- $\mu$ s-long beam pulse with the RFQ fields at the nominal design value. At about $150 \mu$ s into the pulse, the transmission suddenly drops by about $10 \%$. As the RFQ field is increased above the design value, transmission remains high for increasingly longer periods. With fields $\geq 105 \%$ of design, the transmission drop is no longer observed, even for long pulses and $\mathrm{CW}$ operation. Along with the transmission drop, higher-than-expected activation is measured at the high-energy end of the RFQ, indicating significant beam loss at that location. Operating

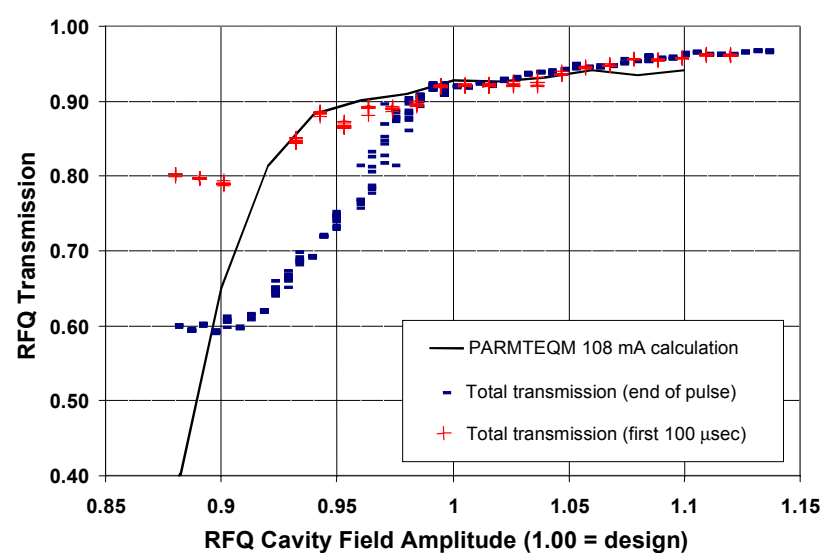

Figure 7: RFQ transmission versus cavity field for a 90-mA, 500- $\mu \mathrm{s}-$ long beam pulse. The anomalous transmission drop occurs at slightly higher fields for increasing beam current.

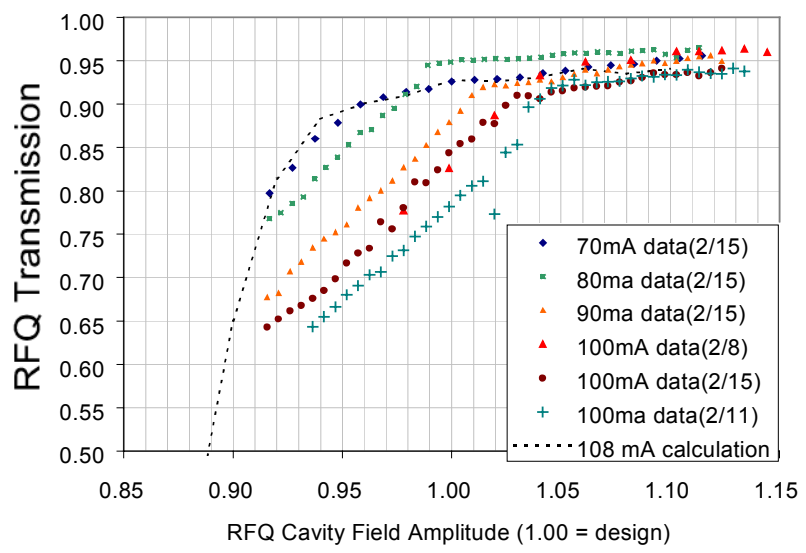

Figure 8: LEDA RFQ transmission vs. cavity field for 70, 80, 90, and $100 \mathrm{~mA}$ RFQ output currents. The PARMTEQM calculation for $108 \mathrm{~mA}$ is also shown.

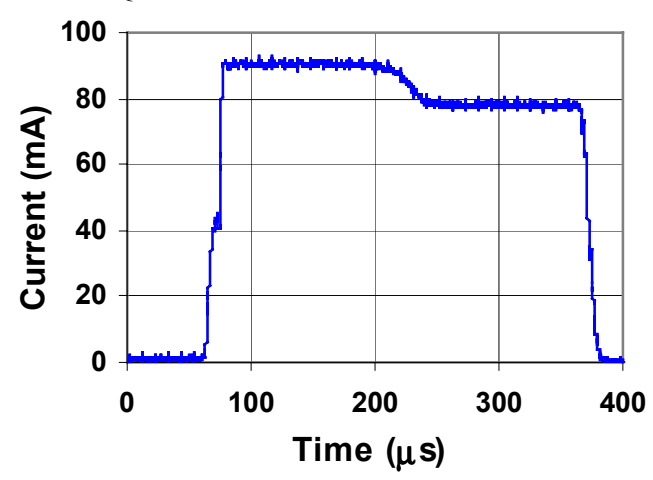

Figure 9: RFQ output beam current vs. time for a 300-mslong pulse at $\sim 97 \%$ of the design RF field level.

the RFQ with fields about $10 \%$ above the design value greatly reduces the magnitude of this beam loss. The total RF power transmitted to the RFQ does not change at the time in the pulse where the transmission drops. However, the RF fields increase slightly in the last meter of the RFQ, as though there were less beam loading in that section [5].

More work is needed to determine unambiguously the cause of the time-dependent transmission anomaly. At 
present, considerable evidence points to the possibility that it may be caused by low-energy $\mathrm{H}^{+}$ions trapped near the axis by the RFQ fields [28]. The extra positive charge from the trapped ions would cause the beam size to increase, reducing the RFQ transmission, and also increasing the beam size at the end of the HEBT. This hypothesis is consistent with the observation that the collimator ring in front of the beam stop glows, presumably from being struck by incident beam, when the RFQ pressure exceeds $1-2 \times 10^{-7}$ Torr. The low-energy $\mathrm{H}^{+}$ ions can be produced by beam collisions with background gas $\left(\mathrm{H}_{2}\right)$ near the RFQ axis, or by beam collision with the vane tip surfaces. At fields $\leq$ the design value, the beam may be sufficiently large that its fringes strike the RFQ vane tips, creating $\mathrm{H}^{+}$ions that get trapped temporarily in the beam channel. As the trapped charge accumulates, the beam becomes larger still, until the transmission drops suddenly.

\section{REFERENCES}

[1] D. Schrage et al.. "CW RFQ Fabrication and Engineering," Proc. LINAC98 (Chicago, 24-28 August 1998) pp. 679683.

[2] L. M. Young et al., "High Power Operations of LEDA," Proc. LINAC2000 (Monterey, 21-25 August 2000) pp. 336-340.

[3] L. M. Young et al., "Low-Energy Demonstration Accelerator (LEDA) Radio-Frequency Quadrupole (RFQ) Results," Proc. 2nd ICFA Advanced Accelerator Workshop on the Physics of High-Brightness Beams (Los Angeles, CA) pp. 488-503.

[4] H. V. Smith, Jr. and J. D. Schneider, "Status Report on the Low-Energy Demonstration Accelerator (LEDA)," Proc. LINAC2000 (Monterey CA, 21-25 August 2000) pp. 581-3.

[5] L. J. Rybarcyk et al., "LEDA Beam Operations Milestone and Observed Beam Transmission Characteristics," Proc. LINAC2000 (Monterey CA, 21-25 August 2000) pp. 584-6.

[6] T. Zaugg, C. Rose, J. D. Schneider, J. Sherman, R. Stevens, Jr., "Operation of a Proton Microwave Source in Pulsed Mode," Proc. LINAC98 (Chicago, 24-28 August 1998) pp. 893-95.

[7] J. D. Sherman et al., "Status Report on a dc 130-mA, 75keV Proton Injector," Rev. Sci. Instrum. 69 (1998) 1003-8.

[8] J. D. Schneider, "Operation of the Low-Energy Demonstration Accelerator: the Proton Injector for APT," Proc. PAC99 (New York, 29 March - 2 April 1999) pp. 503-507.

[9] H. V. Smith, Jr. et al., "Update on the Commissioning of the Low-Energy Demonstration Accelerator (LEDA) Radio-Frequency Quadrupole (RFQ)," Proc. 2nd ICFA Advanced Accelerator Workshop on the Physics of HighBrightness Beams (Los Angeles, CA) pp. 466-479.

[10] D. E. Rees et al., "Design, Operation, and Test Results of $350 \mathrm{MHz}$ LEDA RF System," Proc. LINAC98 (Chicago, 24-28 August 1998) pp. 564- 566.

[11] K. F. Johnson et al., "Commissioning of the Low- Energy Demonstration Accelerator (LEDA) Radio- Frequency Quadrupole (RFQ)," Proc. PAC99 (New York, 29 March 2 April 1999) pp. 3528-3530.
[12] M. J. Browman and L. M. Young, "Coupled RadioFrequency Quadrupoles as Compensated Structures," Proc. of the 1990 Linear Accelerator Conference, (Albuquerque, 10-14 Sept. 1990) LA-12004-C, pp. 70-2.

[13] L. M. Young, "An 8-meter-long Coupled Cavity RFQ Linac," Proc. 1994 Int. Linac Conf., (Tsukuba, 21-26 Aug. 1994) pp. 178-180.

[14] J. H. Billen et al., "A New RF Structure for IntermediateVelocity Particles,” Proc. 1994 Int. Linac Conf. (Tsukuba, 21-26 Aug. 1994) pp. 341-345.

[15] L. M. Young and L. Rybarcyk, "Tuning the LEDA RFQ 6.7 MeV Accelerator," Proc. LINAC98 (Chicago, 24-28 August 1998) pp. 270-272.

[16] K. R. Crandall et al., "RFQ Design Codes," Los Alamos National Laboratory report LA-UR-96-1836 (Revised August 21, 1998).

[17] H. V. Smith, Jr. et al., "Comparison of Simulations with Measurements for the LEDA LEBT H+ Beam," Proc. PAC99 (New York, 29 March - 2 April 1999) pp. 1929-31.

[18] L. M. Young, "PARMELA," Los Alamos National Laboratory report LA-UR-96-1835 (Revised February 27, 2001).

[19] L. M. Young, "Simulations of the LEDA LEBT With H+, H2+, and e- Particles," Proc. of the 1997 Particle Accelerator Conference (Vancouver, 12-16 May 1997) pp. 2749-2751.

[20] L. M. Young, "Simulations of the LEDA RFQ 6.7 MeV Accelerator," Proc. of the 1997 Particle Accelerator Conference (Vancouver, 12-16 May 1997) pp. 2752-2754.

[21] W. D. Kilpatrick, "Criterion for Vacuum Sparking Designed to Include Both rf and dc," Rev. Sci, Instrum. 28, 824 (1957).

[22] M. E. Schulze et al., "Beam Emittance Measurements of the LEDA RFQ," Proc. LINAC2000 (Monterey, 21-25 August 2000) pp. 575-7.

[23] W. P. Lysenko et al., "Determining Phase-Space Properties of the LEDA RFQ Output Beam," Proc. LINAC2000 (Monterey, 21-25 August 2000) pp. 809-11.

[24] G. O. Bolme et al., "Measurement of RF Accelerator Cavity Field Levels at High Power from X-ray Emissions," Proceedings of the 1990 Linear Accelerator Conference, (Albuquerque, 10-14 Sept. 1990) LA-12004-C, pp. 219222.

[25] Robin Ferdinand Joseph Sherman, Ralph R. Stevens, Jr., Thomas Zaugg, "Space-Charge Neutralization Measurement of a 75-Kev, 130-mA Hydrogen-Ion Beam," Proc. PAC97 (Vancouver, B.C., Canada 12-16 May 1997) pp. 27232725.

[26] N. J. Diserens, "Progress in the Development of a 3D Finite Element Computer Program to calculate Space and Image Charge Effects in RF Quadrupoles," IEEE Trans. Nucl. Sci. NS-32 (5), 2501 (1985).

[27] R. Duperrier, R. Ferdinand, J-M, Lagniel, N Pichoff, "Toutatis, The CEA-SACLAY RFQ Code," Proc. LINAC2000 (Monterey, 21-25 August 2000).

[28] M. S. deJong, "Background Ion Trapping in RFQs," Proc. 1984 Linac Conf. (Seeheim, Germany, 7-11 May 1984), pp. 88-90. 